\title{
Penfigoide ocular cicatricial
}

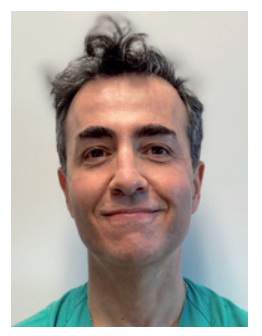

Constantino Jaime Reinoso Montalvo Médico adjunto. Servicio de Oftalmología. Hospital Universitario 12 de Octubre. Madrid.

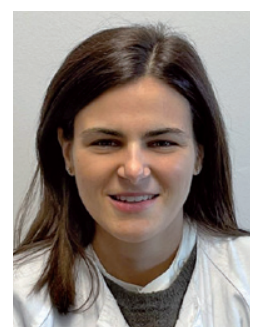

Mónica Gimeno Carrero Médico interno residente. Servicio de Oftalmología. Hospital Universitario 12 de Octubre. Madrid.

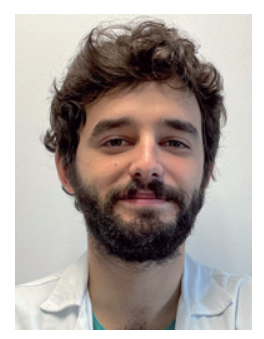

Antonio García Louro Médico interno residente. Servicio de Oftalmología. Hospital Universitario 12 de Octubre. Madrid.

\section{INTRODUCCIÓN}

El penfigoide de las membranas mucosas (PMM) es un fenotipo poco frecuente de enfermedad ampollosa subepidérmica autoinmunitaria, que cursa de forma crónica, caracterizado por la existencia de depósitos lineales de inmunoglobulinas en la membrana basal de las mucosas, con tendencia a la formación de cicatrices, que producen intensa alteración funcional de estas.

El PMM ocular o penfigoide ocular cicatricial (POC) hace referencia al PMM con afectación fundamental de la mucosa conjuntival, que va a dar lugar a una conjuntivitis crónica de tipo cicatricial y que se puede acompañar de inflamación en otras mucosas e, incluso, en zonas de piel sin mucosa.

\section{EPIDEMIOLOGÍA}

La incidencia de esta enfermedad se estima entre 1 de cada 8000 y 1 de cada $50000^{1}$ personas; sin embargo, teniendo en cuenta que las manifestaciones clínicas de las formas leves no se diagnostican en la mayoría de ocasiones como POC, es probable que su incidencia real esté infravalorada.

Suele aparecer en adultos entre 60 y 70 años, siendo rara su presentación en niños. No muestra preferencia por razas o área geográfica, aunque sí parece tener una mayor incidencia en mujeres que en hombres.

\section{FISIOPATOLOGÍA}

El POC es una enfermedad autoinmunitaria sistémica caracterizada por la presencia de autoanticuerpos que actúan mediante una reacción de hipersensibilidad de tipo II frente a estructuras de la membrana basal de la mucosa epitelial. La subunidad $\beta 4$ de la integrina $\alpha 6 \beta 4$ parece desempeñar un papel fundamental en este proceso, siendo el principal autoantígeno.

Se cree que, en toda esta secuencia, tiene un papel importante tanto la genética como el ambiente; así, el alelo HLA-DQ $\beta 1 * 0301$ parece que puede conferir cierta susceptibilidad ${ }^{2}$, así como determinados medicamentos o agentes microbianos que podrían actuar como desencadenantes del proceso inflamatorio.

\section{MANIFESTACIONES CLÍNICAS}

La forma de presentación más habitual es la de una conjuntivitis crónica y recurrente unilateral que progresa hasta afectar a ambos ojos. El proceso continuado y repetido de inflamación y fibrosis conduce a la formación de simbléfaron (adhesiones entre la conjuntiva bulbar y tarsal) (fig. 1) y anquilobléfaron (adhesión entre el margen palpebral del párpado superior e inferior). La cicatrización conjuntival genera, a su vez, la obstrucción de las glándulas lagrimales accesorias, así como la destrucción de células caliciformes, dando lugar a un síndrome de ojo seco (fig. 2). 


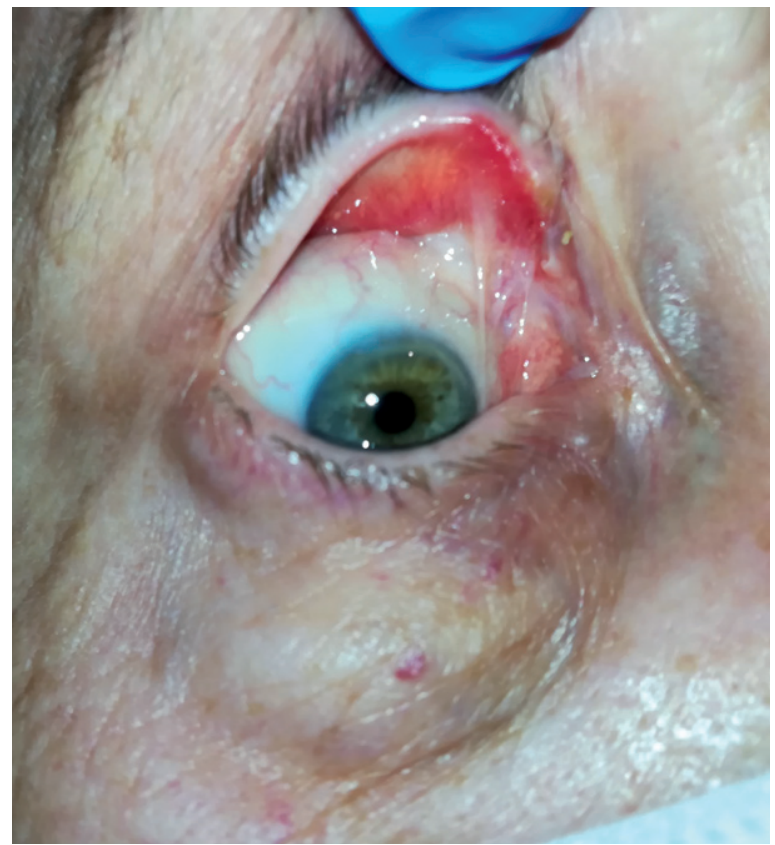

Figura 1. Simbléfaron. Adherencia entre la conjuntiva tarsal superior y bulbar.

Como consecuencia de la fibrosis subepitelial, se produce triquiasis (crecimiento de las pestañas en dirección anómala con el folículo bien posicionado), distriquiasis (metaplasia de las glándulas de Meibomio en los folículos pilosos, produciendo, por lo tanto, más de una hilera de pestañas), entropión (inversión del párpado, generalmente, el inferior, hacia dentro, de tal forma que las pestañas pueden rozar el globo ocular), y lagoftalmos (incapacidad para cerrar completamente los párpados).

Todos los procesos mencionados anteriormente favorecen la queratinización y neovascularización corneal, conduciendo a la pérdida de visión.

\section{CLASIFICACIÓN DE LA GRAVEDAD}

El POC se puede dividir en cuatro estadios en función de la gravedad (clasificación de Foster). El estadio I presenta una conjuntivitis crónica con fibrosis subepitelial; en el II, aparece el acortamiento del fórnix; en el III, simbléfaron; y, final-

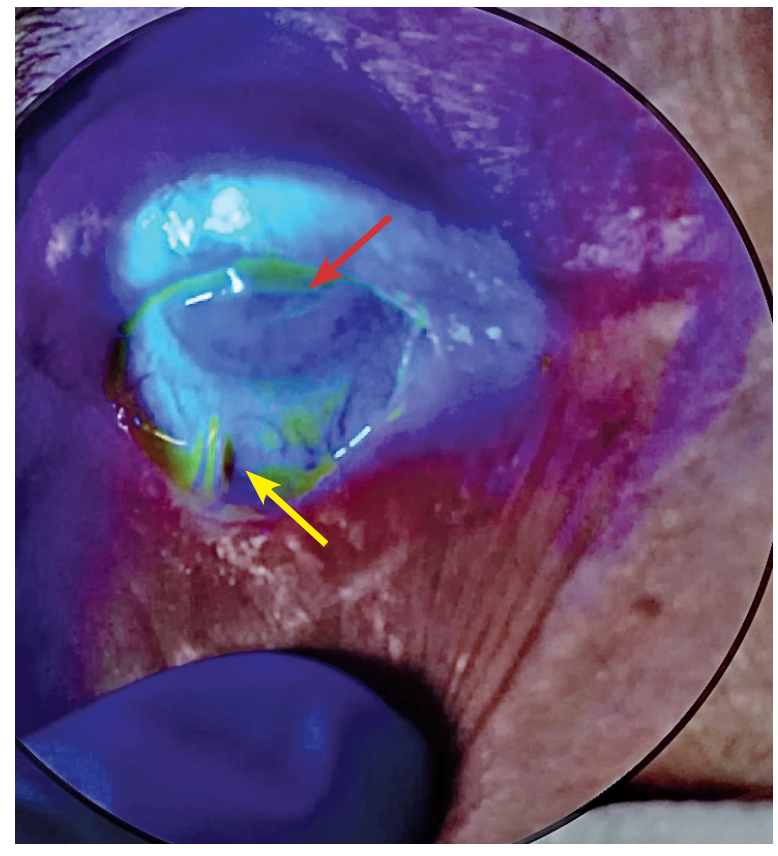

Figura 2. Queratitis punteada superficial (flecha roja) secundaria a ojo seco producido por simbléfaron (fecha amarilla).

mente, en el IV, aparecen anquilobléfaron, síndrome de ojo seco grave y queratinización de la superficie ocular.

Los estadios II y III se subdividen, a su vez, en cuatro grupos en función del grado de afectación expresada en porcentajes ${ }^{1}$ :

- A: $0-25 \%$.

- B: $25-50 \%$.

- C: $50-75 \%$.

- D: $75-100 \%$.

Entre las manifestaciones extraoculares, cabe destacar la afectación oral presente en, aproximadamente, el $40 \%$ de los pacientes, con gingivitis, erosiones y ampollas recidivantes, periodontitis e, incluso, pérdida de piezas dentales. Otras zonas mucosas que pueden verse implicadas son la faringe, la nariz, la laringe, los genitales, el ano y el esófago. También es posible la afectación de la piel sin presencia de mucosa hasta en un $25 \%$ de los casos. 


\section{DIAGNÓSTICO}

La sospecha se origina en la clínica ocular. El diagnóstico de certeza se obtiene mediante biopsia. Para aumentar las posibilidades diagnósticas de la biopsia, se deben tomar las muestras de las zonas que presenten inflamación activa. Ha de tenerse en cuenta que la toma de conjuntiva como muestra puede exacerbar la enfermedad.

No obstante, es conveniente destacar que el resultado negativo en la biopsia no descarta el trastorno $^{1,2}$.

\section{DIAGNÓSTICO DIFERENCIAL}

El diagnóstico diferencial se establece con las otras posibles causas de conjuntivitis cicatricial ${ }^{3}$ (tabla 1).

\section{TRATAMIENTO}

Puede ser médico (local y sistémico) y quirúrgico.

El tratamiento local no tiene un objetivo curativo, puesto que ningún tratamiento tópico ha demostrado ser eficaz frente al POC. Sin embargo, un tratamiento local adecuado frente a la comorbilidad asociada (ojo seco, blefaritis, etc.) supondrá una mejoría importante del malestar del paciente. Entre los tratamientos locales empleados, cabe destacar:

- Lubricantes oculares sin conservantes.

- N-acetilcisteína en caso de queratitis filamentosa.

- Ciclosporina al 0,1 \% y tacrólimus tópico.

- Suero autólogo.

- Calor seco y limpieza del borde palpebral.

El tratamiento sistémico depende de la gravedad:

- Leve:

- Dapsona: es una sulfonamida con propiedades antiinflamatorias. La dosis estándar es de 50-200 mg/día durante, al menos, tres meses.

- Metotrexato: dosis de 5-25 mg/semana.

- Moderado:

- Micofenolato de mofetilo: ha demostrado mayor eficacia frente al POC que los dos anteriores. Es, además, un fármaco bien tolerado.

- Azatioprina.

\section{Tabla 1. Diagnóstico diferencial del penfigoide ocular cicatricial}

\begin{tabular}{l|l}
\hline Enfermedades inflamatorias & $\begin{array}{l}\text { - Blefaroconjuntivitis atópica. } \\
\text { - Rosácea. }\end{array}$ \\
\hline Causas traumáticas & $\begin{array}{l}\text { - Quemaduras térmicas y químicas. } \\
\text { - Traumatismo mecánico. } \\
\text { - Radiación. } \\
\text { - Cirugía. } \\
\text { - Fístula carotidocavernosa. }\end{array}$ \\
\hline Enfermedades autoinmunitarias & $\begin{array}{l}\text { - Síndrome de Stevens-Johnson. } \\
\text { - Enfermedad de injerto contra huésped. }\end{array}$ \\
\hline - Granuloma con poliangitis. \\
- Lupus eritematoso sistémico. \\
- Síndrome de Sjögren.
\end{tabular}


- Grave:

- Ciclofosfamida y prednisona oral: los corticoides orales son útiles para el control agudo de la inflamación en casos graves, pero no son una buena opción como tratamiento a largo plazo por sus numerosos efectos secundarios. Por ello, es fundamental el tratamiento combinado con un ahorrador de corticoides, siendo de elección la ciclofosfamida.

- Inmunoglobulina intravenosa: actualmente, solo se administra si los anteriores no han sido efectivos o el paciente presenta efectos adversos graves.

- Agentes biológicos: anticuerpos dirigidos a los linfocitos B que expresan el clúster de diferenciación 20 (anti CD-20) (rituximab), inhibidores del factor de necrosis tumoral alfa (anti$\mathrm{TNF}-\alpha)^{1}$.

El tratamiento quirúrgico tiene como objetivo reparar aquellas lesiones que provocan daño sobre la superficie ocular (entropión, simbléfaron, triquiasis, etc.). Antes de la realización de la cirugía, es necesario conseguir una estabilidad clínica de la enfermedad para disminuir al mínimo el riesgo de exacerbaciones. Debe evitarse la manipulación de la conjuntiva durante la cirugía, ya que predispone al aumento de la inflamación, con el consiguiente exceso de cicatrización ${ }^{1,3}$. En casos de afectación corneal grave, una de las técnicas más utilizadas es la colocación de membrana amniótica.

\section{CONCLUSIÓN}

El POC es una enfermedad poco común. Por ello, el alto grado de sospecha clínica es fundamental para su diagnóstico y tratamiento temprano, evitando, así, las graves complicaciones que pueden incluso producir ceguera. El dermatólogo a menudo es el primer escalón de la asistencia médica, debiendo integrar rápidamente al oftalmólogo en ella.

El tratamiento médico genera una gran cantidad de efectos adversos. Debido a esto, el diagnóstico ha de ser acertado.

Es necesaria la estabilidad clínica previa a la realización de cirugía para evitar recidivas.

\section{BIBLIOGRAFÍA}

1. Foster S. Ocular cicatricial pemphigoid. Up To Date. 2019. Disponible en: www.uptodate.com/contents/ocular-cicatricialpemphigoid?

2. Valenzuela FA, Perez VL. Mucous membrane pemphigoid. En: Mannis MJ, Holland EJ, (eds.). Cornea: fundamentals, diag-

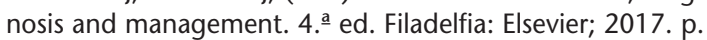
549-57.

3. Kirzhner M, Jakobiec FA. Ocular cicatricial pemphigoid: a review of clinical features, immunopathology, differential diagnosis, and current management. Semin Ophthalmol. 2011; 26(4-5):270-7. 